\title{
Friction Compensation in TP Model Form - Aeroelastic Wing as an Example System
}

\section{Béla Takarics, Péter Baranyi}

Computer and Automation Research Institute

Hungarian Academy of Sciences

Kende u. 13-17, H-1111 Budapest, Hungary

E-mail: takarics@sztaki.hu, baranyi@sztaki.hu

\begin{abstract}
The aim of this paper is to fit the friction compensation problem in the field of modern polytopic and Linear Matrix Inequality (LMI) based control design methodologies. The paper proves that the exact Tensor Product (TP) type polytopic representations of most commonly utilized friction models such as Coulomb, Stribeck and LuGre exist. The paper also determines and evaluates these TP models via a TP model transformation. The conceptual use of the TP model of the friction is demonstrated via a complex control design problem of a $2 D$ aeroelastic wing section. The paper shows how the friction model and the model of the aeroelastic wing section can be merged and transformed to a TP type polytopic model - by TP model transformation - whereupon LMI based control performance optimization can immediately be executed to yield an observer based output feedback control solution to given specifications. The example is evaluated via numerical simulations.
\end{abstract}

Keywords: friction compensation; LMI-based multi-objective control; TP model transformation; qLPV systems

\section{Introduction}

Friction is a highly nonlinear phenomenon and may result in steady state errors, limit cycles, poor performance in high precision applications. Linearizing the friction phenomenon in the control design process can also lead to poor control performance $([1,2])$. There are many general, starting from simple to complex and sophisticated friction models presented in the technical literature given as qLPV (quasi Linear Parameter Varying) models, which can be suitable for inclusion in the model for the controlled plant.

Multi-objective LMI (Linear Matrix Inequality)-based control design for qLPV systems has been in the focus of modern control theories, the pioneers Gahinet, Balas, Chilai, Boyd, and Apkarian were responsible for establishing this new concept 
$[3,4,5]$. The aim of this paper is to fit friction models, and by this friction compensation of qLPV systems to the LMI-based multi-objective control design framework, both theoretically and in practice.

One direction of LMI-based theories needs the system to be formulated in convex polytopic form. The proposed methodology to obtain the convex polytopic form is to apply Tensor Product (TP) model transformation, which is based on the HigherOrder Singular Value Decomposition (HOSVD) of tensors ([6]). The concept of the HOSVD of continuous functions was given in [7] and [8], TP model transformation was introduced to control in [9] as a methodology for system control design.

Various polytopic forms of the same model affect the performance of LMI-based controllers (see $[10,11,12]$ for more information) and TP model transformation is capable of systematic generation of different convex hulls for tensor functions and qLPV models. In this way, the TP model transformation introduces an additional possibility for multi-objective control optimization techniques, namely the convex hull manipulation-based optimization, which is the key property of TP model transformation in control design. The properties of TP model transformation are given below $[13,14,15]$ :

- It can be executed uniformly (irrespective of whether the model is given in an analytical form, as an outcome of soft computing-based identification techniques or as a result of a black-box identification) in a routine fashion without analytical interaction, within a reasonable amount of time.

- It generates the HOSVD-based canonical form of qLPV models. This is a unique representation. This form extracts the unique structure of a given qLPV model in the same sense as the HOSVD does for tensors and matrices, in such a way that: the number of LTI components are minimized; the weighting functions are one variable functions of the parameter vector in an orthonormal system for each parameter; the LTI components are also in orthogonal position; the LTI systems and the weighting functions are ordered according to the higher-order singular values of the parameter vector; unique form.

- TP model transformation was extended to generate different types of convex polytopic models. This was motivated by the fact that the type of convex hull of the polytopic models considerably influences the feasibility of the LMI-based design and the resulting performance. This means that instead of developing new LMI equations for feasible controller design, we may rather focus on the systematic modification of the convex hull $([10,11])$.

- Based on the higher-order singular values (that express the rank properties of the given qLPV model for each element of the parameter vector in $L_{2}$ norm), the TP model transformation offers a trade-off between the complexity of further LMI design and the accuracy of the resulting TP model.

- TP model transformation is executed before utilizing the LMI design. This means that when we start the LMI design we already have the global weight- 
ing functions and during control we do not need to determine a local weighting of the LTI systems for feedback gains.

One can find several applications and related work for TP model transformation in $[16,17,18,19,20,21,22,23,24,25,26,27,28]$. Chumalee et al., Rangajeeva et al., Gai et al., Sun et al. and Qin et al. introduce TP model transformation based novel approaches in avionics related control problems [29, 30, 31, 32], thus leading to pioneering conceptual frameworks.

The first step in fitting friction compensation to LMI-based multi-objective control design methodology is to check whether the most commonly used friction models can be defined by a finite element polytopic form. The second step is to bring friction phenomenon and friction models to the same polytopic structure with the other elements of the control system, which makes LMI-based multi-objective control design methodology available for friction compensation in the same conceptual level.

The paper is organized as follows: Section 2 gives a brief introduction to the commonly used friction models, Section 3 introduces the proposed methodology to transform friction models to polytopic form and LMI-based multi-objective control design. Section 4 lists the polytopic form of the commonly used friction models and Section 5 deals with the friction compensation of an example dynamic system, namely 2 degrees-of-freedom aeroelastic wing section. The conclusions finish the paper.

\section{Commonly Used Friction Models}

Friction is a physical phenomenon and expressed in quantitative terms as a force $F_{f}$, being the force exerted by either of two contacting bodies tending to oppose relative tangential displacement of the other [33]. The following friction models are commonly used in the control of mechatronic systems.

Definition 1 (Coulomb friction force): The Coulomb friction force is a force of constant magnitude, acting in the direction opposite to motion $v(t)$. When $v(t) \neq 0$ :

$$
F_{f}(t)=-F_{c} \operatorname{sign}(v(t)), \quad F_{c}(t)=\mu F_{n},
$$

where $F_{n}$ is the normal component of the force pressing surfaces together and $\mu$ is the frictional coefficient. $\mu$ is determined by measurements under certain conditions.

Definition 2 (Viscous friction): The viscous friction models the friction force as a force proportional to sliding velocity. When $v(t) \neq 0$ :

$$
F_{f}(t)=-F_{v} v(t)
$$


where $F_{v}$ is the coefficient of viscous friction.

Definition 3 (Static friction): The static friction is the influence of an external force for the friction at rest, however, this leads to a discontinuous function.

Definition 4 (The Stribeck model) The Stribeck friction model is defined by the following equation, when $v(t) \neq 0$ [34]:

$$
F_{f}(t)=-\left(F_{c}+\left(F_{s}-F_{c}\right) e^{-\left|\frac{v}{v_{s}}\right|^{\delta}}\right) \operatorname{sign}(v(t))-F_{v} v,
$$

where $v_{s}$ is the Stribeck velocity, $\delta$ is an empirical parameter, $F_{s}$ is the static friction.

Definition 5 (The LuGre model) The LuGre model is a dynamic friction model presented in [35]. Friction is modeled as the average deflection force of elastic springs. When a tangential force is applied the bristles will deflect like springs. If the deflection is sufficiently large enough, the bristles start to slip. The LuGre model has the form

$$
\frac{d z}{d t}=v-\delta_{0} \frac{|v|}{g(v)} z, \quad F=\delta_{0} z+\delta_{1}(v) \frac{d z}{d t}+f(v),
$$

where $z$ denotes the average bristle deflection. The parameter $\delta_{0}$ is the stiffness of the bristles, and $\delta_{1}(v)$ the damping. The function $g(v)$ models the Stribeck effect, and $f(v)$ the viscous friction. A reasonable choice of $g(v)$, which gives a good approximation of the Stribeck effect, is $g(v)=\alpha_{0}+\alpha_{1} e^{-\left(v / v_{0}\right)^{2}}$. The sum $\alpha_{0}+\alpha_{1}$ then corresponds to stiction force and $\alpha_{0}$ to Coulomb friction force. The parameter $v_{0}$ determines how $g(v)$ varies within its bounds $\alpha_{0}<g(v) \leq \alpha_{0}+\alpha_{1}$. A common choice of $f(v)$ is linear viscous friction $f(v)=\alpha_{2} v$. The main properties of the LuGre friction model are also listed in [36], who also applied the model.

\section{TP Model Transformation}

Assume we have the qLPV model as:

Definition 6 (qLPV model): Consider the quasi Linear Parameter Varying State Space model:

$$
\begin{aligned}
\dot{\mathbf{x}}(t) & =\mathbf{A}(\mathbf{p}(t)) \mathbf{x}(t)+\mathbf{B}(\mathbf{p}(t)) \mathbf{u}(t) \\
\mathbf{y}(t) & =\mathbf{C}(\mathbf{p}(t)) \mathbf{x}(t)+\mathbf{D}(\mathbf{p}(t)) \mathbf{u}(t),
\end{aligned}
$$

with input $\mathbf{u}(t) \in \mathbb{R}^{m}$, output $\mathbf{y}(t) \in \mathbb{R}^{l}$ and state vector $\mathbf{x}(t) \in \mathbb{R}^{k}$. The system matrix

$$
\mathbf{S}(\mathbf{p}(t))=\left(\begin{array}{ll}
\mathbf{A}(\mathbf{p}(t)) & \mathbf{B}(\mathbf{p}(t)) \\
\mathbf{C}(\mathbf{p}(t)) & \mathbf{D}(\mathbf{p}(t))
\end{array}\right)
$$


is a parameter-varying object, where $\mathbf{p}(t) \in \Omega$ is time varying $N$-dimensional parameter vector, where $\Omega=\left[a_{1}, b_{1}\right] \times\left[a_{2}, b_{2}\right] \times . . \times\left[a_{N}, b_{N}\right] \in \mathbb{R}^{N}$ is a closed hypercube. $\mathbf{p}(t)$ can also include some elements of $\mathbf{x}(t)$, in this case (6) is termed as quasi LPV (qLPV) model and as such, it belongs to the class of non-linear models.

Definition 7 (Finite element TP type polytopic model): $\mathbf{S}(\mathbf{p}(t))$ in (5) is given for any parameter as the parameter-varying combination of LTI system matrices $\mathbf{S}_{r} \in \mathbb{R}^{(m+k) \times(m+k)}$ as:

$$
\mathbf{S}(\mathbf{p}(t))=S \stackrel{\bigotimes_{n=1}^{N}}{\mathbf{w}}\left(p_{n}(t)\right)
$$

by applying the compact notation based on tensor algebra (Lathauwer's work [6]), where the $(N+2)$ dimensional coefficient tensor $\mathbf{S} \in \mathbb{R}^{I_{1} \times I_{2} \times \cdots \times I_{n} \times(m+k) \times(m+k)}$ is constructed from the LTI vertex systems $\mathbf{S}_{i_{1}, i_{2}, \ldots, i_{N}}(7)$ and the row vector $w_{n}\left(p_{n}(t)\right) \in[0,1]$ contains one variable and continuous weighting functions $w_{n, i_{n}}\left(p_{n}(t)\right),\left(i_{n}=1 \ldots I_{N}\right)$.

Definition 8 (Convex type TP model): The TP model is SN (Sum Normalized) if the sum of the weighting functions for all $p \in \Omega$ is 1 and it is $N N$ (Non-Negative) if the values of the weighting functions for all $p \in \Omega$ are non-negative. The TP model is convex if it is SNNN:

$$
\forall n, i, p_{n}(t): w_{n, i}\left(p_{n}(t)\right) \in[0,1] ; \quad \forall n, p_{n}(t): \sum_{i=1}^{I_{n}} w_{n, i}\left(p_{n}(t)\right)=1
$$

It was shown in $[10,37]$ that the type of the convex hull has a large influence on the feasibility and control performance of the LMI-based convex optimalization, therefore, various types of convex hulls are defined [38]:

Definition 9 (NO/CNO, NOrmal type TP model): The convex TP model is a NO (normal) type model, if its $w(p)$ weighting functions are Normal, that is, if it satisfies (8) and the largest value of all weighting functions is 1 . Also, it is CNO (close to normal), if it is satisfies (8) and the largest value of all weighting functions is 1 or close to 1.

Definition 10 (IRNO, Inverted and Relaxed NOrmal type TP model): The TP model is IRNO type if the smallest values of all weighting functions are 0 , and the largest values of all weighting functions are the same.

The basic definitions and the steps of TP model transformation are also given in $[37,13,39,40,41,42,43]$. 


\subsection{Steps of TP Model Transformation}

The paper presents a TP model transformation of functions describing nonlinear friction models. Therefore, the steps of TP model transformation of functions are given, which can be generalized for transforming qLPV systems to convex polytopic form.

\section{Step 1: Discretization.}

The goal of this step is to represent the given function $(y=f(\mathbf{x}))$ or qLPV model $(\mathbf{S}(\mathbf{p}(t)))$ by its discretized tensor $\mathscr{F} D(\Omega, M)$.

We define the transformation space $\Omega$ in which we expect the TP function to be relevant and the hyper-rectangular grid $M$. As a result of discretizing the function $y=f(\mathbf{x})$ over the grid points we have $\mathscr{F} D(\Omega, M) \in \mathbb{R}^{M_{1} \times \ldots \times M_{N}}$.

Step 2: Extracting the discretized TP function.

The goal of this step is to reveal the TP structure of the given function. We use HOSVD to find the TP structure of the function.

As a result of STEP 1 , we have the discretized system tensor $\mathscr{F} D(\Omega, M)$. The CHOSVD results in $\mathscr{F}^{D(\Omega, M)}$ :

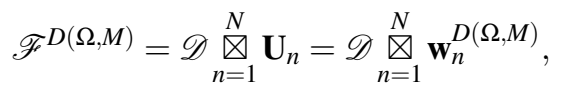

where the size of $\mathscr{D}$ is $R_{1} \times R_{2} \times \ldots \times R_{N}$. Since $R_{n}=\operatorname{rank}_{n}\left(\mathscr{F}^{D(\Omega, M)}\right), R_{n} \leq$ $M_{n}$, for all $n=1 . . N$. Tensor $\mathscr{D}$ contains the constant components $\mathbf{D}_{i_{1}, i_{2}, \ldots, i_{N}}$, $i_{n}=1 . . I_{n}$. Based on the previous section, we have $\mathbf{U}_{n}=\mathbf{w}_{n}^{D(\Omega, M)}$.

Step 3: Reconstruction of the continuous TP function.

The weighting functions can be determined over any points of intervals $\left[a_{n}, b_{n}\right]$ by the help of the given $y=f(\mathbf{x})$. In order to determine the weighting functions in vector $\mathbf{w}_{d}\left(x_{d}\right)$, let $x_{n}$ be fixed to selected grid-lines as:

$$
x_{n}=g_{n, i_{n}} \quad n=1 \ldots N, \quad n \neq d, \quad i_{n} \in\left\{1 \ldots I_{n}\right\},
$$

where $i_{n}$ can be chosen arbitrarily. Then for $x_{d}$ :

$$
\mathbf{w}_{d}\left(x_{d}\right)=(\mathbf{y}(\mathbf{x}))_{(3)}\left(\left(\mathscr{B} \underset{n \neq d}{\underset{n}{\otimes} \mathbf{u}_{n, i_{n}}}\right)_{(d)}\right)^{+},
$$

where vector $\mathbf{x}$ consists of elements $x_{n}$ and $x_{d}$ as $\mathbf{x}=\left(g_{1, i_{1}} g_{2, i_{2}} \ldots p_{d} \ldots g_{N, i_{n}}\right)$, and superscript "+" denotes pseudo inverse and $\mathbf{u}_{n, i_{n}}$ is the first row vector of $\mathbf{U}_{n}$. The third-mode matrix $(\mathbf{y}(\mathbf{x}))_{(3)}$ of function $\mathbf{y}(\mathbf{x})$ is understood such that function $\mathbf{y}(\mathbf{x})$ is considered as a three-dimensional tensor, where the length of the third-dimension is one. This in practice means that the function $\mathbf{y}(\mathbf{x})$ is stored in a one-row vector. 


\section{TP Type Polytopic Form of Some Commonly Used Friction Models}

\subsection{TP Type Polytopic Form of the Coulomb Friction Model}

The Coulomb friction in Definition 1 is described by (1).

Due to finite precision of numerical calculations it is necessary to involve an arbitrary parameter to handle the sharp transition between $v=0$ and $v \neq 0$. A solution is to approximate the signum function in the following way:

$$
\operatorname{sign}(v)=\frac{2}{\left(1+e^{-1000 v}\right)}-1,
$$

where exponent 1000 can be arbitrarily set. The value does not influence the structure of the model, only the slope of the resulting weighting functions.

Based on Definition 1 and the approximation of the signum function as in (11), we can execute the TP model transformation on the Coulomb friction given in the following form:

$$
F_{\text {Coulomb }}=f(v)=-F_{c}\left(\frac{2}{\left(1+e^{-1000 v}\right)}-1\right) .
$$

The aim is to give the TP type polytopic form of the Coulomb friction as:

$$
F_{\text {Coulomb }}(v)=\mathscr{B} \bigotimes_{n=1}^{N} \mathbf{w}_{n}(v),
$$

where $F_{\text {Coulomb }}$ is scalar and $v \in \mathbb{R}, \mathscr{B} \in \mathbb{R}^{I_{1} \times \ldots \times I_{N}}$ is bounded and the row vector $\mathbf{w}_{n}(v) \in[0,1]$ contains one variable and continuous weighting functions $w_{n, i_{n}}(v)$, $\left(i_{n}=1 \ldots I_{N}\right)$. As a first step of the TP model transformation we have to define the transformation space $\Omega$. We expect the model to be valid in speed region from $a$ $\mathrm{m} / \mathrm{s}$ to $b \mathrm{~m} / \mathrm{s}$. Therefore, we define the transformation space as $\Omega=[a, b]$ (note that these intervals can be arbitrarily defined). TP model transformation starts with the discretization over a rectangular grid. Let the density of the discretization grid be $M$.

\subsubsection{Convex Type TP Models of the Coulomb Friction}

LMI-based multi objective controller design requires the friction model $F_{f}(v)$ to be given in a multi-dimensional convex hull, thus we construct various multidimensional convex hulls of $F_{f}(v)$ from the HOVSD-based canonical form of the Coulomb friction. 
Definition 11 (Convex model of the Coulomb friction): By applying convexity criteria ( $S N N N$ type convex hull), we get that the Coulomb friction $F_{f}(v)$ of $(12)$ can be reconstructed from 2 LTI vertex systems as:

$$
F_{\text {Coulomb }}(v)=\sum_{i=1}^{2} w_{i}^{\text {snnn }}(v) B_{i}^{\text {snnn }}=\mathscr{B}_{\text {snnn }}{\underset{n=1}{\otimes}}_{n}^{N} \mathbf{w}_{n_{\text {snnn }}}(v) .
$$

Definition 12 (CNO type convex model of the Coulomb friction): The CNO type convex hull represents a tight hull, which is advantageous for controller design.

$$
F_{\text {Coulomb }}(v)=\sum_{i=1}^{2} w_{i}^{\text {cno }}(v) B_{i}^{\text {cno }}=\mathscr{B}_{\text {cno }} \underset{n=1}{N} \mathbf{w}_{n_{\text {cno }}}(v) .
$$

Definition 13 (Convex model of the Coulomb friction): The IRNO type convex hull represents a large convex hull.

$$
F_{\text {Coulomb }}(v)=\sum_{i=1}^{2} w_{i}^{\text {irno }}(v) B_{i}^{\text {irno }}=\mathscr{B}_{\text {irno }} \underset{n=1}{\otimes} \mathbf{w}_{n_{\text {irno }}}(v) .
$$

The weighting functions of the canonical form and different convex type TP models of an academic Coulomb friction are given in Figure 1.

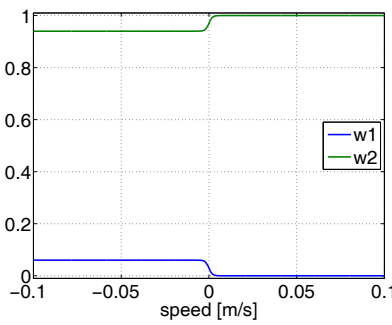

(a)

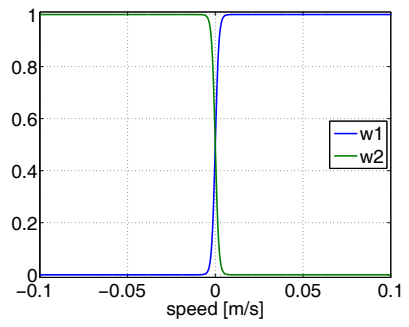

(b)

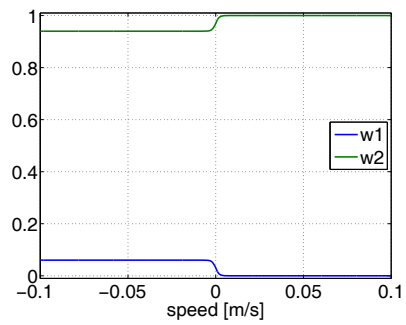

(c)

Figure 1

Weighting functions of the Coulomb friction model a) SNNN type convex model; (b) CNO type convex model; (c) IRNO type convex model;

\subsection{TP Type Polytopic Form of the Coulomb and Viscous Friction Model}

Based on Definition 2 and the approximation of the signum function as in (11), we can execute the TP model transformation on the viscous and Coulomb friction model given in the following form: 


$$
F_{f}=f(v)=-F_{c}\left(\frac{2}{\left(1+e^{-1000 v}\right)}-1\right)-F_{v} v
$$

The aim is to give the TP type polytopic form of the viscous and Coulomb friction as:

$$
F_{\text {Coulomb }+ \text { viscous }}(v)=\mathscr{B} \bigotimes_{n=1}^{N} \mathbf{w}_{n}(v),
$$

where $F_{\text {Coulomb }+ \text { viscous }}$ is scalar and $v \in \mathbb{R}, \mathscr{B} \in \mathbb{R}^{I_{1} \times \ldots \times I_{N}}$ is bounded and the row vector $\mathbf{w}_{n}(v) \in[0,1]$ contains one variable and continuous weighting functions $w_{n, i_{n}}(v)$ ,$\left(i_{n}=1 \ldots I_{N}\right)$.

\subsection{TP Type Polytopic Form of the Stribeck Friction Model}

Based on Definition 4 and the approximation of the signum function as in (11), we can execute the TP model transformation on the Stribeck friction model given as:

$$
F_{f}=f(v)=-\left(F_{c}+\frac{\left(F_{s}-F_{c}\right)}{\left(1+\left(\frac{v}{v_{s}}\right)^{2}\right)}\right)\left(\frac{2}{\left(1+e^{-1000 v}\right)}-1\right)-F_{v} v
$$

The aim is to give the TP type polytopic form of the Stribeck friction as:

$$
F_{\text {Stribeck }}=\mathscr{B} \stackrel{\bigotimes}{n=1}_{\mathbb{N}}^{N} \mathbf{w}_{n}(v),
$$

where $F_{\text {Stribeck }}$ is scalar and $v \in \mathbb{R}, \mathscr{B} \in \mathbb{R}^{I_{1} \times \ldots \times I_{N}}$ is bounded and the row vector $\mathbf{w}_{n}(v) \in[0,1]$ contains one variable and continuous weighting functions $w_{n, i_{n}}(v)$, $\left(i_{n}=1 \ldots I_{N}\right)$. The weighting functions can be seen in Figure 2 .

\subsection{TP Type Polytopic Form of the LuGre Friction Model}

Based on Definition 5, we can give the LuGre friction in a form suitable for TP model transformation as:

$$
F_{f}=f(\mathbf{x})=\delta_{0} z+\delta_{1}\left(v-\frac{\delta_{0}|v|}{F_{c}+\left(F_{s}-F_{c}\right) e^{-\left|v / v_{s}\right|}} z\right)+\delta_{2} v .
$$

The aim is to give the TP type polytopic form of the LuGre friction as: 


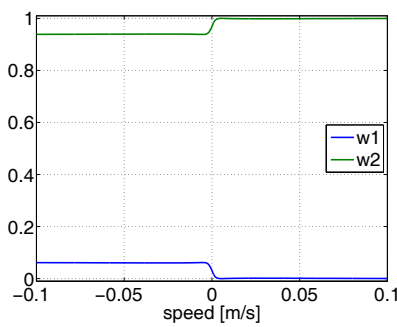

(a)

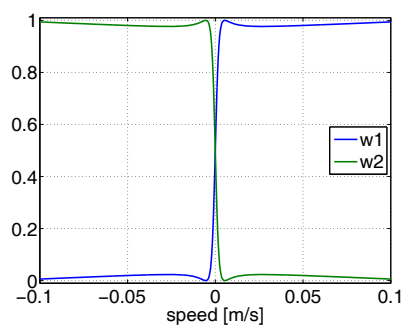

(b)

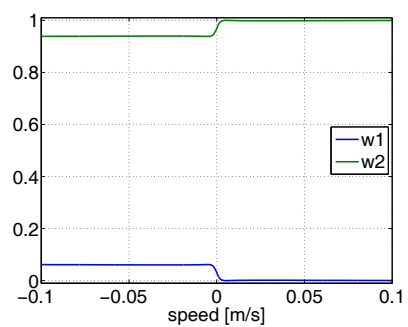

(c)
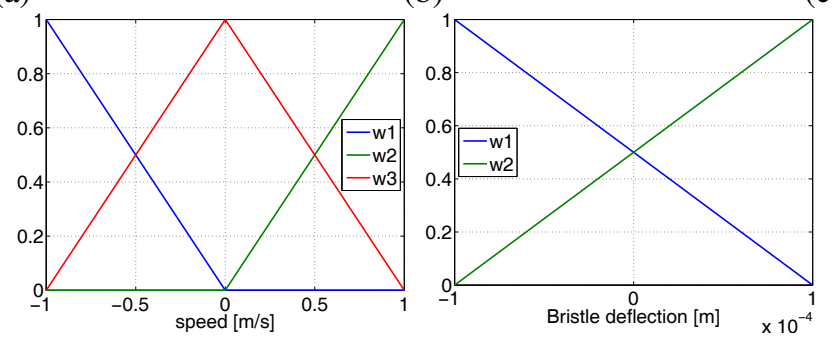

(d)

Figure 2

Weighting functions of the Stribeck and LuGre friction modela a) SNNN type convex Stribeck model; (b) CNO type convex Stribeck model; (c) IRNO type convex Stribeck model; (d) CNO type convex LuGre model

$$
F_{\text {LuGre }}=\mathscr{B} \underset{n=1}{N} \mathbf{w}_{n}(\mathbf{x}),
$$

where $F_{\text {LuGre }}$ is scalar and $\mathbf{x} \in \mathbb{R}^{2}, \mathscr{B} \in \mathbb{R}^{I_{1} \times \ldots \times I_{N}}$ is bounded and the row vector $\mathbf{w}_{n}(\mathbf{x}) \in[0,1]$ contains one variable and continuous weighting functions $w_{n, i_{n}}(\mathbf{x})$, $\left(i_{n}=1 \ldots I_{N}\right)$. Vector $\mathbf{x}$ contains the velocity $v$ and $z$, which denotes the average bristle deflection. $F_{c}$ denotes the Coulomb friction, $F_{S}$ the static friction, $v_{s}$ the Stribeck velocity, $\delta_{0}$ the stiffness of the bristles, $\delta_{1}$ the microdamping and $\delta_{2}$ the viscous friction. We expect the model to be valid in a speed region from $a_{1} \mathrm{~m} / \mathrm{s}$ to $b_{1} \mathrm{~m} / \mathrm{s}$ and average bristle deflection from $a_{2} m$ to $b_{2} \mathrm{~m}$. After executing the steps of TP model transformation we get the convex polytopic form of the LuGre friction, which can be exactly reconstructed with minimum 6 LTI vertex models as:

$$
F_{\text {LuGre }}(v, z)=\sum_{i=1}^{3} \sum_{j=1}^{2} w_{1, i}(v) w_{2, j}(z) B_{i, j}=\mathscr{B} \underset{n=1}{\bigotimes} \mathbf{w}_{n}\left(p_{n}(t)\right)
$$

where $B_{i, j}$ contains the vertex points of tensor $\mathscr{B} \in \mathbb{R}^{3 \times 2}$. The same types of convex hull can also be constructed for the LuGre model, Figure 2 shows the weighting functions of the CNO type convex form. 


\section{Example Friction Compensation: 2 DoF Aeroelastic Wing Section}

\section{1 qLPV Model of the Aeroelastic Wing with Nonlinear Friction}

The aeroelastic wing model with nonlinear friction was validated by experiments in [44]. Similar models with linear friction were used in [45] [46], [47] and [48]. The parameters and the equations of motion with qLPV representation of the 2 DoF prototypical aeroelastic wing section are given in [47]. The difference between the present qLPV model and the qLPV model given in [47] lies in the friction. Authors have reported discrepancies between the experimental measurements and the analytical analysis [49]. These discrepancies can most likely be accounted for in the Coulomb damping forces that occur within the pitch bearing and plunge slider motion that is not taken into account in many of the models [50]. The equations for the plunge and pitch damping forces are as follows:

$$
F_{h}=\mu_{h} m g|\dot{h}| \dot{h}^{-1}, \quad F_{\alpha}=\mu_{\alpha} M_{f}|\dot{\alpha}| \dot{\alpha}^{-1}
$$

$M_{f}$ is the frictional moment due to the nonlinear cam and $\mu_{h}, \mu_{\alpha}$ are the frictional coefficients. Equation (24) is identical to (1), thus we apply the Coulomb friction from Definition 1. The parameters of the friction models are given in [50].

\subsection{Execution of TP Model Transformation}

First of all we define the transformation space $\Omega:[14,25] \times[-0.1,0.1] \times[-2.5,2.5]$ $\times[-2.5,2.5]$ in which we expect the TP model be relevant, then we discretize the qLPV model in $M_{1} \times M_{2} \times M_{3} \times M_{4}$ points, where $M_{1}=M_{2}=M_{3}=M_{4}=102$.

In this paper we generate the exact minimized form, this means that we eliminate only the zero singular values. The number of non-zero singular values on the first dimension is 3 and on the second, third and fourth dimension is 2 . The numerical values are the following respectively: 519297, 196869, 401.154, 469459, 296706, $555361,1009.52,555361$ and 51.7953. We found that the model can be exactly given in TP type polytopic form with the combination of 24 LTI vertex systems. The CNO type weighting functions can be seen in Figure 3.

\subsection{Controller and Observer Design for the Aeroelastic Wing with Nonlinear Friction}

LMI based multi-objective, robust control design can be executed immediately on convex TP type polytopic models [9]. Therefore, the aim is to transform the friction models to convex polytopic form via TP model transformation. An LTI feedback 

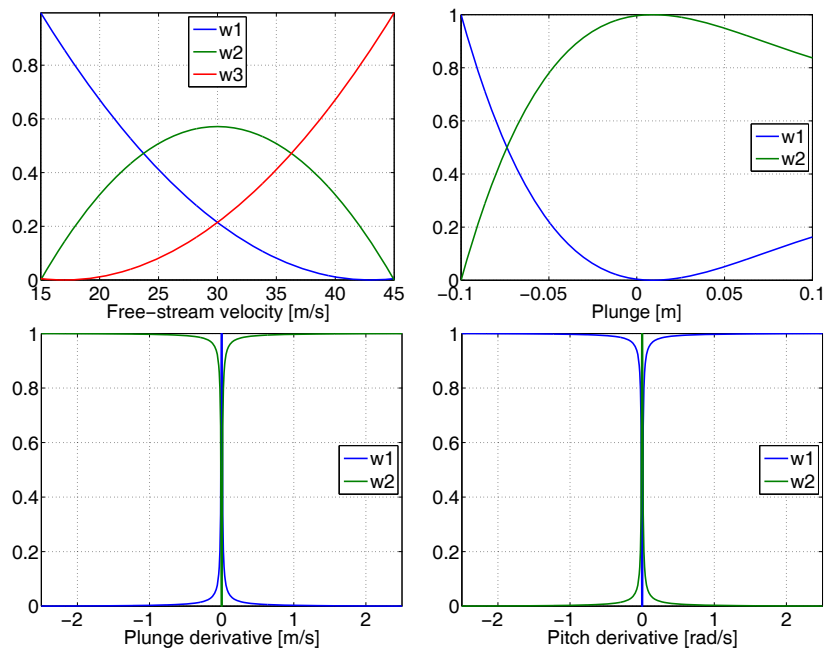

Figure 3

CNO type weighting functions of the aeroelastic model with nonlinear friction

gain is determined for each LTI vertex system of a given convex TP model based on feasibility test of the selected LMIs. In practical applications the state of the system is often not readily available. In this case one has to define the polytopic observer structure. There are various alternative ways to achieve output feedback and observer design (in this regard it is referred to [51, 52]). The observers are required to satisfy the following:

$$
\mathbf{x}(t)-\hat{\mathbf{x}}(t) \rightarrow 0 \quad \text { as } \quad t \rightarrow \infty,
$$

where $\hat{\mathbf{x}}(t)$ denotes the state-vector estimated by the observer. This condition guarantees that the steady-state error between $\mathbf{x}(t)$ and $\hat{\mathbf{x}}(t)$ converges to 0 . The present case is restricted to systems in which $\mathbf{p}(t)$ does not contain values form the estimated state-vector $\hat{\mathbf{x}}(t)$. The following strategy for controller and observed design can be used in this case:

$$
\begin{aligned}
\hat{\mathbf{x}}(t) & =\mathbf{A}(\mathbf{p}(t)) \hat{\mathbf{x}}(t)+\mathbf{B}(\mathbf{p}(t)) \mathbf{u}(t)+\mathbf{K}(\mathbf{p}(t))(\mathbf{y}(t)-\hat{\mathbf{y}}(t)) \\
\hat{\mathbf{y}}(t) & =\mathbf{C}(\mathbf{p}(t)) \hat{\mathbf{x}}(t) .
\end{aligned}
$$

The polytopic model form of this structure is: 


$$
\begin{aligned}
\hat{\mathbf{x}}(t)= & \mathscr{A} \bigotimes_{n=1}^{N} \mathbf{w}_{n}\left(p_{n}(t)\right) \hat{\mathbf{x}}(t)+\mathscr{B} \bigotimes_{n=1}^{N} \mathbf{w}_{n}\left(p_{n}(t)\right) \mathbf{u}(t)+ \\
& +\mathscr{K} \bigotimes_{n=1}^{N} \mathbf{w}_{n}\left(p_{n}(t)\right)_{r}(\mathbf{y}(t)-\hat{\mathbf{y}}(t)) \\
\hat{\mathbf{y}}(t)= & \mathscr{C} \bigotimes_{n=1}^{N} \mathbf{w}_{n}\left(p_{n}(t)\right) \hat{\mathbf{x}}(t) .
\end{aligned}
$$

The goal of the controller and observer design is to determine gains $\mathscr{F}$ and $\mathscr{K}$ in such a way that the stability of the observer and the controller is simultaneously guaranteed. The control signal in output feedback control gets the following form:

$$
u(t)=-\mathscr{F} \bigotimes_{n=1}^{N} \mathbf{w}_{n}\left(p_{n}(t)\right) \hat{\mathbf{x}}(t)
$$

There are several LMI theorems available for observer and controller design to derive the vertex gains $\mathscr{K}$ of the observer and the feedback gains $\mathscr{F}$ of the controller. The feasibility test of the following LMI was executed in the example case:

Theorem 1 (Globally and asymptotically stable observer and controller with decay rate) Based on LMIs presented in [52] one can derive the following LMI:

$$
\begin{aligned}
\mathbf{P}_{1} \mathbf{A}_{r}^{T}-\mathbf{M}_{1, r}^{T} \mathbf{B}_{r}^{T}+\mathbf{A}_{r} \mathbf{P}_{1}-\mathbf{B}_{r} \mathbf{M}_{1, r}+2 \alpha \mathbf{P}_{1} & <\mathbf{0}, \\
\mathbf{A}_{r}^{T} \mathbf{P}_{2}-\mathbf{C}_{r}^{T} \mathbf{N}_{2, r}^{T}+\mathbf{P}_{2} \mathbf{A}_{r}-\mathbf{N}_{2, r} \mathbf{C}_{r}+2 \alpha \mathbf{P}_{2} & <\mathbf{0}, \\
\mathbf{P}_{1} \mathbf{A}_{r}^{T}-\mathbf{B}_{s} \mathbf{M}_{1, r}-\mathbf{M}_{1, s}^{T} \mathbf{B}_{r}^{T}+\mathbf{A}_{s} \mathbf{P}_{1}-\mathbf{B}_{r} \mathbf{M}_{1, s}+\mathbf{P}_{1} \mathbf{A}_{s}^{T}-\mathbf{M}_{1, r}^{T} \mathbf{B}_{s}^{T}+\mathbf{A}_{s} \mathbf{P}_{1}+4 \alpha & \\
\mathbf{P}_{1} & <\mathbf{0}, \\
\mathbf{A}_{r}^{T} \mathbf{P}_{2}-\mathbf{C}_{s}^{T} \mathbf{N}_{2, r}^{T}+\mathbf{P}_{2} \mathbf{A}_{r}-\mathbf{N}_{2, r} \mathbf{C}_{s}+\mathbf{A}_{s}^{T} \mathbf{P}_{2}-\mathbf{C}_{r}^{T} \mathbf{N}_{2, s}^{T}+\mathbf{P}_{2} \mathbf{A}_{s}-\mathbf{N}_{2, s} \mathbf{C}_{r}+4 \alpha \mathbf{P}_{2} & <\mathbf{0},
\end{aligned}
$$

which leads to an asymptotically stable observer and controller decay rate.

One can apply further LMIs in order to guarantee various additional constraints.

Remark 1 Convex hull manipulation is also an important step of the optimization, but it is out of the scope of this paper. More information about convex hull manipulation in general and with a special focus on the aeroelastic wing section model can be found in [10].

The aim of the controller is to stabilize the $2 \mathrm{DoF}$ prototypical aeroelastic wing section in the flutter velocity range. The controller has to satisfy the following criteria: The closed-loop system is stable (in this case we aim at achieving asymptotic stability); The closed-loop system exhibits good settling behavior for a class of initial conditions. The aeroelastic model was transformed to finite element convex TP 
model form in the previous section, upon which LMI-based control design can immediately be executed. In the present case $U$, the plunge deflection $\left(x_{1}(t)\right)$ and pitch angle $\left(x_{2}(t)\right)$ are measurable, but the unavailable state values $x_{3}(t)$ and $x_{4}(t)$ have to be estimated. Solving LMIs of Theorem 1 simultaneously leads to the control and observer gains in the form of $\mathscr{F}$ and $\mathscr{K}$ respectively. As mentioned earlier, convex hull manipulation is an important optimization technique (see [10] for more details). Since it is out of scope of the paper, based on the results of [10] we conclude that the best hull type for our case is the CNO type (see Definition 9).

\subsection{Validation of the Controller Design}

The numerical examples are performed with $a=-0.4$ and with free stream velocity $U=20 \mathrm{~m} / \mathrm{s}$, a velocity which exceeds the linear flutter velocity $U=15.5 \mathrm{~m} / \mathrm{s}$, and for initials $h=0.01 \mathrm{~m}$ and $\alpha=0.1 \mathrm{rad}$. Figure 4 shows the time response of the controlled system. An important issue should be addressed here. Theorem 1 claims that the resulting controller is globally stable. However, the TP-model transformation is a numerical method that can be performed over an arbitrary, but bounded, domain $\Omega$. Therefore, the global stability, ensured by Theorem 1 , is restricted to $\Omega$ and as such it is termed quasi global stability. This, however, has practical significance because the accuracy of a given model is also bounded in reality. In the present example the prototypical aeroelastic model is accurate only for low speeds, and we have defined $\Omega$ accordingly in the design process. The performance of the controller is evaluated based on the following aspects: settling time of the pitch $-0.2 \mathrm{~s}$; number of oscillations of the pitch - no oscillation; maximal overshot of the pitch - no overshot; settling time of the plunge - $0.75 \mathrm{~s}$; number of oscillations of the plunge - 4; maximal overshot of the plunge - $110 \%$ of the original error; maximal torque of the controller - $52 \mathrm{Nm}$. Comparing the control results one can observe that the controllers developed in this paper are considerable faster than in [50]. We can notice that the friction compensation is well managed, the controller results are similar as in $[47,53]$, where the same controller design method was utilized but the aeroelastic model lacks nonlinear friction.
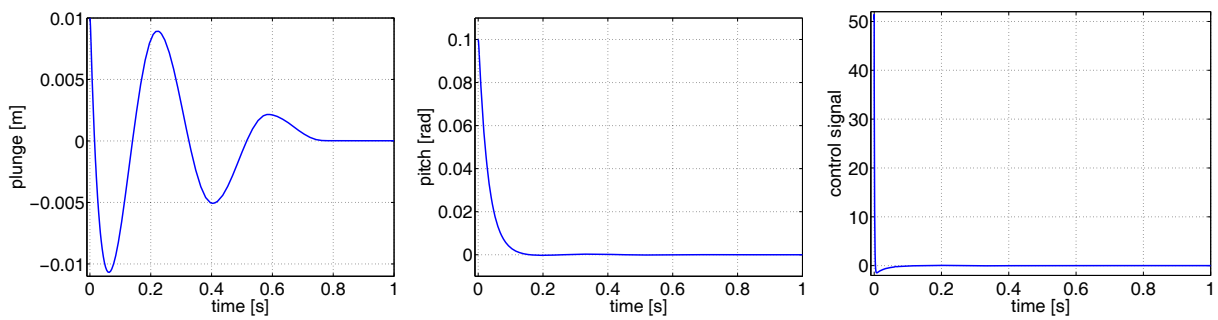

Figure 4

Time response of the designed controller for $U=20 \mathrm{~m} / \mathrm{s}$ and $a=-0.4$ 


\section{Conclusions}

The most commonly used friction models were transformed to a convex polytopic form via TP model transformation. In this way, friction compensation can is fit to a LMI-based, modern and multi-objective control design. All of the presented friction models can be decomposed to a finite element polytopic form.

The extension of qLPV systems with nonlinear friction models increases the number of the dimension of the system. However, extending the qLPV system with the presented friction model does not lead to explosion in the number of necessary vertex systems in the polytopic form and preserves the exact control design, which is the main theoretical result of the paper.

The effectiveness of the proposed friction compensation methodology was presented through the qLPV model of an aeroelastic wing section with Coulomb friction. The qLPV model was transformed to TP type polytopic model on which LMI based controllers can be designed. The polytopic decomposition of such a model is possible and the model can be exactly described by 24 LTI vertex points. The designed controller and observer was validated by simulation and it was compared to the controllers of other authors, dealing with the same problem. We can say that the designed controller performance is on par with the controller designed for the system with linear damping and it is faster than the controllers for nonlinear damping found in the current literature.

\section{Acknowledgement}

The research was supported by the National Research and Technology Agency, (ERC_09) (OMFB-01677/2009) (ERC-HU-09-1-2009-0004 MTA SZTAKI) and Control Research Group of Hungarian Academy of Science.

\section{References}

[1] C. C. de Wit, H. Olsson, K. J. Astrom, and P. Lischinsky, "Dynamic friction models and control design," in In Proceedings of the American Control Conference, 1993, pp. 1920-1926.

[2] H. Olsson, Control Systems with Friction. PhD thesis. Lund Institute of Technology, University of Lund, 1996.

[3] P. Apkarian and P. Gahinet, "A convex characterization of gain-scheduled $\mathrm{H}_{\infty}$ controllers," IEEE Trans. Aut. Contr., 1995.

[4] S. Boyd, L. E. Ghaoui, E. Feron, and V. Balakrishnan, Linear Matrix Inequalities in Systems and Control Theory. Philadelphia: SIAM books, 1994.

[5] J. Bokor and G. Balas, "Linear matrix inequalities in systems and control theory," in Perprints of 16th IFAC Word Congress, 2005, p. Keynote lecture. 
[6] L. D. Lathauwer, B. D. Moor, and J. Vandewalle, "A multi linear singular value decomposition," SIAM Journal on Matrix Analysis and Applications, vol. 21, no. 4, pp. 1253-1278, 2000.

[7] P. Baranyi, L. Szeidl, P. Várlaki, and Y. Yam, "Definition of the HOSVD-based canonical form of polytopic dynamic models," in 3rd International Conference on Mechatronics (ICM 2006), Budapest, Hungary, July 3-5 2006, pp. 660-665.

[8] _ "Numerical reconstruction of the HOSVD-based canonical form of polytopic dynamic models," in 10th International Conference on Intelligent Engineering Systems, London, UK, June 26-28 2006, pp. 196-201.

[9] P. Baranyi, "TP model transformation as a way to LMI based controller design," IEEE Transaction on Industrial Electronics, vol. 51, no. 2, April 2004.

[10] P. K. P. Grof and P. Baranyi, "Different determination of the stability parameter space of a two dimensional aeroelastic system, a tp model based approach," IEEE 14th International Conference on Intelligent Engineering Systems, pp. 0-6, 2010.

[11] P. Baranyi, "Output feedback control of two-dimensional aeroelastic system," Journal of Guidance, Control, and Dynamics, vol. 29, no. 3, pp. 762-767, MayJune 2005.

[12] P. Grof, P. Galambos, and P. Baranyi, "Convex hull manipulation based control performance optimization: Case study of impedance model with feedback delay.” IEEE, Jan. 2012, pp. 495-499.

[13] Z. Petres, "Polytopic decomposition of linear parameter-varying models by tensor-product model transformation," Ph.D. dissertation, Budapest, Hungary, November 2006.

[14] S. Nagy, P. Baranyi, and Z. Petres, "Centralized tensor product model form." IEEE, Jan. 2008, pp. 189-193.

[15] L. Szeidl and P. Varlaki, "Hosvd based canonical form for polytopic models of dynamic systems," Journal of Advanced Computational Intelligence and Intelligent Informatics, vol. 13, no. 1, pp. 52-60, Jan. 2009.

[16] R. Precup, L. Dioanca, E. M. Petriu, M. Radac, S. Preitl, and C. Dragos, "Tensor product-based real-time control of the liquid levels in a three tank system," in 2010 IEEE/ASME International Conference on Advanced Intelligent Mechatronics, Montreal, QC, Canada, jul 2010, pp. 768-773.

[17] Z. Szabó, P. Gáspár, and J. Bokor, "A novel control-oriented multi-affine qLPV modeling framework," in Control Automation (MED), 2010 18th Mediterranean Conference on, Jun. 2010, pp. 1019-1024. 
[18] C. Sun, Y. Huang, C. Qian, and L. Wang, "On modeling and control of a flexible air-breathing hypersonic vehicle based on LPV method," Frontiers of Electrical and Electronic Engineering, vol. 7, no. 1, pp. 56-68, 2012.

[19] T. Luspay, T. Péni, and B. Kulcsar, "Constrained freeway traffic control via linear parameter varying paradigms," Control of Linear Parameter Varying Systems With Applications, p. 461, 2012.

[20] P. Korondi, "Sector sliding mode design based on tensor product model transformation," in Intelligent Engineering Systems, 2007. INES 2007. 11th International Conference on, Jul. 2007, pp. 253-258.

[21] R. Precup, C. Dragos, S. Preitl, M. Radac, and E. M. Petriu, "Novel tensor product models for automatic transmission system control," IEEE Systems Journal, p. In print., 2012.

[22] S. Ilea, J. Matusko, and F. Kolonic, "Tensor product transformation based speed control of permanent magnet synchronous motor drives," in 17th International Conference on Electrical Drives and Power Electronics, EDPE 2011 (5th Joint Slovak-Croatian Conference), 2011.

[23] B. Takarics and P. Baranyi, "TP Model-based Robust Stabilization of the 3 Degrees-of-Freedom Aeroelastic Wing Section," Acta Polytechnica Hungarica, vol. 12, no. 1, Feb. 2014.

[24] P. Baranyi, "TP model transformation as a manipulation tool for qLPV analysis and design," Asian Journal of Control, vol. 17, no. 2, pp. 497-507, Mar. 2015.

[25] J. Kuti, P. Galambos, and Á. Miklós, "Output feedback control of a dualexcenter vibration actuator via qLPV model and TP model transformation," Asian Journal of Control, vol. 17, no. 2, pp. 432-442, Mar. 2015.

[26] S. Chumalee and J. F. Whidborne, "Gain-scheduled $H_{\infty}$ control for tensor product type polytopic plants," Asian Journal of Control, vol. 17, no. 2, pp. 417-431, Mar. 2015.

[27] R.-E. Precup, E. M. Petriu, M.-B. R?dac, S. Preitl, L.-O. Fedorovici, and C.A. Drago?, "Cascade control system-based cost effective combination of tensor product model transformation and fuzzy control," Asian Journal of Control, vol. 17, no. 2, pp. 381-391, Mar. 2015.

[28] T. T. Wang, W. F. Xie, G. D. Liu, and Y. M. Zhao, "Quasi-Min-Max model predictive control for image-based visual servoing with tensor product model transformation," Asian Journal of Control, vol. 17, no. 2, pp. 402-416, Mar. 2015 .

[29] S. Chumalee and J. Whidborne, LPVAutopilot Design of a Jindivik UAV. American Institute of Aeronautics and Astronautics, 1801 Alexander Bell Dr., Suite 500 Reston VA 20191-4344 USA,, 2009. 
[30] W. Qin, Z. Zheng, G. Liu, J. Ma, and W. Li, "Robust variable gain control for hypersonic vehicles based on LPV," Systems Engineering and Electronics, vol. 33, no. 6, pp. 1327-1331, 2011.

[31] S. Rangajeeva and J. Whidborne, "Linear parameter varying control of a quadrotor," in Industrial and Information Systems (ICIIS), 2011 6th IEEE International Conference on, Aug. 2011, pp. 483-488.

[32] W. Gai, H. Wang, T. Guo, and D. Li, "Modeling and LPV flight control of the canard rotor/ wing unmanned aerial vehicle," in Artificial Intelligence, Management Science and Electronic Commerce (AIMSEC), 2011 2nd International Conference on, Aug. 2011, pp. 2187-2191.

[33] P. Korondi, P. Bartal, and F. Kolonic, "Friction model based on tensor product transformation," in 7th International Symposium of Hungarian Researchers on Computational Intelligence, Budapest, Budapest, 2006, pp. 83-94.

[34] D. P. Hess and A. Soom, "Friction at a lubricated line contact operating at oscillating sliding velocities," Journal of Tribology, vol. 112, no. 1, pp. 147152, 1990.

[35] C. C. de Wit, H. Olsson, K. J. Astrom, and P. Lischinsky, "A new model for control of systems with friction," IEEE Transactions on Automatic Control, vol. 40, no. 3, pp. 419-425, 1995.

[36] P. J. Dolcini, "Contribution to the clutch comfort," Ph.D. dissertation, Grenoble, France, May 2007.

[37] P. Gróf, P. Baranyi, and P. Korondi, "Convex hull manipulation based control performance optimisation,” WSEAS Trans. Sys. Ctrl., vol. 5, no. 8, pp. 691-700, Aug. 2010.

[38] P. Baranyi, "Convex hull generation methods for polytopic representations of LPV models.” IEEE, Jan. 2009, pp. 69-74.

[39] P. Z. Baranyi and Y. Yam, "Uniform observer design for linear parameter varying systems," in Computational Intelligence. Proceedings of the 5th International Symposium of Hungarian Researchers, Budapest. Budapest: BME, 2004, pp. 371-381.

[40] B. Takarics, "Parallel distributed compensation based sector sliding mode control of takagi-sugeno type polytopic models." IEEE, Jan. 2012, pp. 501-506.

[41] Z. Petres, B. Resko, and P. Baranyi, "Reference signal control of the TORA system: a TP model transformation based approach,” vol. 2. IEEE, Jul. 2004, pp. 1081-1086.

[42] Z. Petres and P. Z. Baranyi, "Approximation and complexity trade-off by tp model transformation in controller design: a case study of the tora system," 
in 8th international symposium of Hungarian researchers on computational intelligence and informatics. Magyar kutatók 8. nemzetközi szimpóziuma. Budapest, 2007., Budapest, 2007, pp. 441-455.

[43] P. Galambos, P. Z. Baranyi, and P. Korondi, "Extended tp model transformation for polytopic representation of impedance model with feedback delay," WSEAS Transactions on Systems and Control, vol. 5, no. 9, pp. 701-710, September 2010 .

[44] J. J. Block and T. W. Strganac, "Applied active control for nonlinear aeroelastic structure," Journal of Guidance, Control, and Dynamics, vol. 21, no. 6, pp. 838845, November-December 1998.

[45] Y. C. Fung, An Introduction to the Theory of Aeroelasticity. John Wiley and Sons, New York, 1955.

[46] E. H. D. (Editor), H. C. J. Curtiss, R. H. Scanlan, and F. Sisto, A Modern Course in Aeroelasticity. Stifthoff and Noordhoff, Alpen aan den Rijn, The Netherlands, 1978.

[47] P. Baranyi, "Tensor product model based control of 2-d aeroelastic system," Journal of Guidance, Control, and Dynamics, vol. 29, no. 2, March-April 2006.

[48] P. Marzocca and L. Librescu, "Aeroelastic response of nonlinear wing sections using a functional series technique," AIAA Journal, vol. 40, no. 5, May 2002.

[49] R. Duvignequ and M. Visonneau, "Optimization of a synthetic jet actuator for aerodynamic stall control," Laboratorire de Mecanique des Fluides CNRS UMR 6598, 2005.

[50] J. Block and H. Gilliatt, "Active control of an aeroelastic structure," AIAA Meeting Papers on Disc, 1997.

[51] C. W. Scherer and S. Weiland, Linear Matrix Iequalities in Control, ser. DISC course lecture notes, DOWNLOAD: http://www.cs.ele.tue.nl/SWeiland/lmid.pdf, 2000 .

[52] K. Tanaka and H. O. Wang, Fuzzy Control Systems Design and Analysis - A Linear Matrix Inequality Approach. Hohn Wiley and Sons, Inc., 2001, 2001.

[53] P. Baranyi, P. Korondi, and H. Hashimoto, "Global asymptotic stabilization of the prototypical aeroelastic wing section via TP model transformation," Asian Journal of Control, vol. 7, no. 2, pp. 99-111, Oct. 2008. 